Research Article

\title{
Postulation of General Unions of Lines and Multiplicity Two Points in $\mathbb{P}^{3}$
}

\author{
E. Ballico \\ Department of Mathematics, University of Trento, 38123 Povo, Italy \\ Correspondence should be addressed to E. Ballico; ballico@science.unitn.it
}

Received 3 January 2014; Accepted 11 February 2014; Published 23 March 2014

Academic Editors: D. Franco and A. Simis

Copyright (C) 2014 E. Ballico. This is an open access article distributed under the Creative Commons Attribution License, which permits unrestricted use, distribution, and reproduction in any medium, provided the original work is properly cited.

We prove that a general union $X \subset \mathbb{P}^{3}$ of prescribed numbers of lines and double points has maximal rank, except a few well-known exceptional cases.

\section{Introduction}

Fix $P \in \mathbb{P}^{r}$. The 2-point $2 P$ of $\mathbb{P}^{r}$ is the closed subscheme of $\mathbb{P}^{r}$ with $\left(\mathscr{I}_{P}\right)^{2}$ as its ideal sheaf. The scheme $2 P$ is a zerodimensional scheme with $\operatorname{deg}(2 P)=r+1$ and $(2 P)_{\text {red }}=\{P\}$. Now assume that $r \geq 3$. For all $(t, a) \in \mathbb{N}^{2}$, let $Z(r, t, a)$ be the set of all disjoint unions $X \subset \mathbb{P}^{r}$ of $t$ lines and $a$ 2-points. Assume that $(t, a) \neq(0,0)$; that is, assume that $Z(r, t, a) \neq \emptyset$. For each $X \in Z(r, t, a)$ and each integer $x \geq 0$, we have $h^{0}\left(\mathcal{O}_{X}(x)\right)=(x+1) t+a(r+1)$. The algebraic set $Z(r, t, a)$ is an irreducible subset of the Hilbert scheme. We recall that a closed subscheme $W \subset \mathbb{P}^{r}$ is said to have maximal rank if for each integer $x \geq 0$, the restriction map $H^{0}\left(\mathcal{O}_{\mathbb{P}^{r}}(x)\right) \rightarrow$ $H^{0}\left(\mathcal{O}_{X}(x)\right)$ is a linear map with maximal rank; that is, it is either injective or surjective. $W$ has maximal rank if and only if for each integer $x \geq 0$ either $h^{0}\left(\mathscr{I}_{W}(x)\right)=0$ or $h^{1}\left(\mathcal{O}_{X}(x)\right)=$ 0 . In this paper we prove the following result.

Theorem 1. Fix $(t, a) \in \mathbb{N}^{2} \backslash\{(0,0)\}$ and an integer $k \geq 3$. Let $X$ be a general element of $Z(3, t, a)$. Then either $h^{0}\left(\mathscr{I}_{X}(k)\right)=0$ $\operatorname{orh}^{1}\left(\mathscr{J}_{X}(k)\right)=0$, unless either $(t, a, k)=(2,3,3)$ or $(t, a, k)=$ $(0,9,4)$.

The case $(t, a, k)=(0,9,4)$ is one of the exceptional cases in the famous Alexander-Hirschowitz theorem [1-5]. See $[6$, Example 1] for the case $(t, a, k)=(2,3,3)$. Theorem 1 is obviously false for $k=2$, but it is false in a controlled way [6, Lemma 1], because a disjoint union of 3 lines of $\mathbb{P}^{3}$ is contained in a unique quadric surface and for each $P \in \mathbb{P}^{3}$, the linear system $\left|\mathscr{I}_{2 P}(2)\right|$ is the set of all quadric cones with vertex containing $P$. Therefore as an immediate corollary of Theorem 1, we get the following result.

Corollary 2. Fix $(t, a) \in \mathbb{P}^{3}$ such that $t+a \geq 4,(t, a) \neq(2,3)$, and $(t, a) \neq(0,9)$. Let $X$ be a general element of $Z(3, t, a)$. Then $X$ has maximal rank.

The case $k=3,4,5$ of Theorem 1 was proved in [6, Propositions 1,2,3] and a weaker form of Theorem 1 was proved in [6, Proposition 5] (we required that $(k+1) t+4 a+$ $\left.3 k \leq\left(\begin{array}{c}k+3 \\ 3\end{array}\right)\right)$. This weaker version was enough to prove the statement corresponding to Theorem 1 first in $\mathbb{P}^{4}$ and then in $\mathbb{P}^{5}$. Then we proved by induction on $r$ the corresponding statement in $\mathbb{P}^{r}, r \geq 5$, but only if $k \geq 4$ [7].

For the proof, we use the case $a=0$ proved by Hartshorne and Hirschowitz [8] and the case $t=0$, that is, the AlexanderHirschowitz theorem $[1,2,4,5]$. We use certain nilpotent structures on reducible conics (called sundials in [9]) and on lines (called +lines in [10]; see Section 2 for them).

Our interest in this topic (after, of course, $[1-3,8,11]$ ) was reborn by Carlini et al. who started a long project about the Hilbert functions of multiple structures on unions of linear subspaces of $\mathbb{P}^{r}[9,12,13]$.

\section{Preliminary Lemmas}

Let $\mathrm{Q} \subset \mathbb{P}^{3}$ be a smooth quadric surface. For each finite set $S \subset \mathbb{P}^{3}$, set $2 S:=\cup_{P \in S} 2 P$. For each closed subscheme $X \subset \mathbb{P}^{3}$, 
the residual scheme $\operatorname{Res}_{Q}(X)$ of $X$ with respect to $Q$ is the closed subscheme of $X$ with $\mathscr{I}_{X}: \mathscr{I}_{\mathrm{Q}}$ as its ideal sheaf. For each integer $x$, we have the following exact sequence (often called Castelnuovo's sequence):

$$
0 \longrightarrow \mathscr{I}_{\operatorname{Res}_{Q}(X)}(x-2) \longrightarrow \mathscr{I}_{X}(x) \longrightarrow \mathscr{I}_{X \cap Q}(x) \longrightarrow 0
$$

From (1) we get $h^{0}\left(\mathscr{I}_{X}(x)\right) \leq h^{0}\left(\mathscr{I}_{\operatorname{Res}_{Q}(X)}(x-2)\right)+$ $h^{0}\left(Q, \mathscr{I}_{X \cap Q}(x)\right)$ and $h^{1}\left(\mathscr{I}_{X}(x)\right) \leq h^{1}\left(\mathscr{I}_{\operatorname{Res}_{Q}(X)}(x-2)\right)+$ $h^{1}\left(Q, \mathscr{I}_{X \cap Q}(x)\right)$. Let $Z \subset Q$ be a zero-dimensional subscheme, and let $E \subset Q$ be a union of $y$ distinct lines of type $(0,1)$. The residual scheme $\operatorname{Res}_{E}(Z)$ of $Z$ is the closed subscheme of $Q$ with $\mathscr{I}_{Z}: \mathscr{I}_{E}$ as its ideal sheaf. For each $x \in \mathbb{Z}$, we have Castelnuovo's exact sequence of coherent sheaves on $Q$ :

$$
0 \longrightarrow \mathscr{I}_{\operatorname{Res}_{E}(Z)}(x, x-y) \longrightarrow \mathscr{I}_{Z}(x, x) \longrightarrow \mathscr{I}_{E \cap Z, E}(x, x)
$$

$\longrightarrow 0$.

Hence $h^{i}\left(Q, \mathscr{I}_{Z}(x, x)\right) \leq h^{i}\left(Q, \mathscr{I}_{\operatorname{Res}_{E}(Z)}(x, x-y)\right)+$ $h^{i}\left(E, \mathscr{I}_{Z \cap E}(x, x)\right), i=0,1$. We have $h^{1}\left(E, \mathscr{I}_{Z \cap E}(x, x)\right)=0$ if and only if $\operatorname{deg}(Z \cap L) \leq x+1$ for each connected component $L$ of $E$.

Let $Z(3, t, a)^{\prime}$ denote the closure of $Z(3, t, a)$ in the Hilbert scheme of $\mathbb{P}^{3}$. Fix a line $L \subset \mathbb{P}^{3}$ with $P \in L$ and a line $D \subset \mathbb{P}^{3}$ with $P \in D$ and $D \neq L$. Set $v:=2 P \cap D$. Set $X:=L \cup v$ (it is the intersection of $L \cup D$ with the scheme $L \cup 2 P$ ). As in [10], we call $X$ a +line with $L$ as its support and $P$ as the support of its nilradical.

For all $(x, y) \in \mathbb{N}^{2} \backslash\{(0,0)\}$, let $L(3, x, y)$ be the set of all disjoint unions $X \subset \mathbb{P}^{3}$ of $x$ lines and $y+$ lines. The algebraic set $L(3, x, y)$ is irreducible and its general element has maximal rank [10, Theorem 1]. For all integers $(x, y) \in \mathbb{N}^{2}$, let $L(3, x, y)_{\mathrm{Q}}$ denote the set of all $X \in L(3, x, y)$ such that $\operatorname{dim}(X \cap Q)=0$ and the support of the nilradical of $\mathcal{O}_{X}$ is contained in $Q$. The algebraic set $L(3, x, y)_{\mathrm{Q}}$ is irreducible. For a general $X \in L(3, x, y)$, we have $\operatorname{Res}_{Q}(X)=X$; that is, for each point $P$ in the support of the nilradical of $\sigma_{X}$, the tangent vector $v$ representing the nilradical of $\mathcal{O}_{X}$ is not tangent to $Q$.

For all integers $k \geq 0$, set $a_{k}:=\lfloor(k+3)(k+2) / 6\rfloor, b_{k}:=$ $\left(\begin{array}{c}k+3 \\ 3\end{array}\right)-(k+1) a_{k}$, and $z_{k}:=\lfloor(k+3)(k+2) / 6\rfloor-\lfloor(k+1) k / 6\rfloor$. We have $a_{k}=(k+3)(k+2) / 6$ and $b_{k}=0$ if $k \equiv 0,1(\bmod 3)$ and $a_{k}=(k+3)(k+2) / 6-1 / 3, b_{k}=(k+1) / 3$ if $k \equiv 2(\bmod 3)$. We have $z_{6 t}=4 t+1, z_{6 t+1}=4 t+2, t>1, z_{6 t+2}=4 t+2$, $z_{6 t+3}=4 t+3, z_{6 t+4}=4 t+4$, and $z_{6 t+5}=4 t+4$.

For each integer $x \geq 0$, let $D_{3 x+2}^{\prime}$ and $D_{3 x+2}^{\prime \prime}$ denote the following assertions.

Assertion $D_{3 x+2}^{\prime}: h^{i}\left(\mathscr{I}_{X}(k)\right)=0, i=0,1$, for a general $X \in L(3,\lfloor(k+3)(k+2) / 6\rfloor,(k+1) / 3)_{\mathrm{Q}}$.

Assertion $D_{3 x+2}^{\prime \prime}: h^{i}\left(\mathscr{I}_{X}(k)\right)=0, i=0,1$, for a general $X \in L(3,\lfloor(k+3)(k+2) / 6\rfloor-1,4(k+1) / 3)_{\mathrm{Q}}$.

Remark 3. Since 9 general points of $\mathbb{P}^{3}$ are contained in a smooth quadric and a general $X \in L(3,\lfloor(k+3)(k+2) / 6\rfloor,(k+$ $1) / 3$ ) has maximal rank [10, Theorem 1$], D_{3 x+2}^{\prime}$ is true if $x+1 \leq$
9 , that is, if $x \leq 10$. For the same reason, $D_{3 x+2}^{\prime \prime}$ is true if $4 x+3 \leq 9$, that is, if $x \leq 1$.

Lemma 4. $D_{3 x+2}^{\prime}$ is true for all $x \geq 0$.

Proof. Fix a general $Y \in L\left(3, a_{3 x}, 0\right)$. We have $h^{i}\left(\mathscr{I}_{Y}(3 x)\right)=0$, $i=0,1$, and $Y \cap Q$ is a general union of $(x+1)(3 x+2)$ points. Let $E \subset Q$ be a general union of $z_{3 x+2}=2 x+2$ lines of type $(0,1)$. Fix $S \subset Y \cap Q$ with $\sharp(S)=x+1$ and $\sharp(S \cap L) \leq 1$ for each line $L \subset Y$. Write $Y=Y^{\prime} \sqcup Y^{\prime \prime}$ with $Y^{\prime \prime}$ the union of the lines of $Y$ containing a point of $S$. Let $G \subset \mathbb{P}^{3}$ be a disjoint union of $x+1+$ lines with $Y^{\prime \prime}=G_{\text {red }}$, and let $S$ be the support of the nilradical of $G$ and with $Q$ containing each tangent vector of a +lines of $G$; that is, we assume that $\operatorname{deg}(G \cap Q)=3(x+1)$ and that $\operatorname{Res}_{Q}(G)=Y^{\prime \prime}$. Set $X:=Y^{\prime} \cup E \cup G$. Deforming $E$ to a general union of $z_{3 x+2}$ lines of $\mathbb{P}^{3}$, we get that $X$ is a flat limit of a family of elements of $\mathrm{L}\left(3, \mathrm{a}_{3 \mathrm{x}+2}-\mathrm{X}-1, \mathrm{X}+1\right)_{\mathrm{Q}}$. By the semicontinuity theorem for cohomology [14, III.12.8], it is sufficient to prove that $\mathrm{h}^{\mathrm{i}}\left(\mathscr{I}_{\mathrm{X}}(\mathrm{k})\right)=0, \mathrm{i}=0,1$. Since $\operatorname{Res}_{\mathrm{Q}}(\mathrm{X})=\mathrm{Y}$, it is sufficient to prove that $h^{i}\left(\mathrm{Q}, \mathscr{I}_{\mathrm{Q} \cap X}(k)\right)=$ $0, i=0,1$; that is, $h^{i}\left(Q, \mathscr{I}_{\operatorname{Res}_{E}(X \cap Q)}(3 x+2, x)\right)=0$. These equalities are true, because $\operatorname{Res}_{E}(X \cap Q)$ is the union of $2 a_{3 x}-$ $2 x-2$ general points and $x+1$ general tangent vectors of $Q$ and $2(x+1) \leq(3 x+2)+x$; in characteristic zero we may also quote two general results, that is, [15] (for general zerodimensional curvilinear schemes) and [16, Lemma 1.4] (for general tangent vectors).

For each integer $k \geq 2$, let $D_{k}$ denote the following assertion.

Assertion $D_{k}, k \geq 2$ : for every integer $y$ such that $0 \leq y \leq$ $k+1$, we have $h^{1}\left(\mathscr{I}_{X}(k)\right)=0$ for a general $X \in L\left(3, a_{k}-1-\right.$ $y, y)_{\mathrm{Q}}$.

Remark 5. Fix an integer $k \geq 2$ and assume the existence of $Y \in L\left(3, a_{k}-k-2, k+1\right)_{\mathrm{Q}}$ such that $h^{1}\left(\mathscr{I}_{Y}(k)\right)=0$. Since $h^{0}\left(\mathcal{O}_{Y}(k)\right)=\left(\begin{array}{c}k+3 \\ 3\end{array}\right)-b_{k}$, we have $h^{0}\left(\mathscr{I}_{Y}(k)\right)=b_{k}$. Fix an integer $y$ such that $0 \leq y \leq k$. Take any $X \in L\left(3, a_{k}-1-y, y\right)$ such that $X \subset Y$. Obviously, $X \in L\left(3, a_{k}-1-y, y\right)_{\mathrm{Q}}$, $h^{1}\left(\mathscr{I}_{X}(k)\right)=0$, and $h^{0}\left(\mathscr{I}_{X}(k)\right)=b_{k}+k+1-y$. Therefore to prove $D_{k}$, it is sufficient to prove its case " $y=k+1$."

Lemma 6. $D_{k}$ is true for all $k \geq 2$ and $D_{3 x+2}^{\prime \prime}$ is true for all $x \geq 0$.

Proof. By Remark 5 to prove $D_{k}$, we may assume that $k \geq 9$ and only check the case " $y=k+1$ " of $D_{k}$.

(a) Assume that $k \equiv 2(\bmod 3)$. Fix a general $Y \in$ $L\left(3, a_{k-2}, 0\right)$. We have $h^{i}\left(\mathscr{I}_{Y}(k-2)\right)=0, i=0,1[8]$, and $Y \cap Q$ is a general union of $2 a_{k-2}$ points of $Q$. Fix $S \subset Y \cap Q$ such that $\sharp(S)=k+1$ and each line of $Y$ contains at most one point of $S$. Write $Y=Y^{\prime} \sqcup Y^{\prime \prime}$ with $Y^{\prime \prime}$ the union of the components of $Y$ containing a point of $S$. Let $G \subset \mathbb{P}^{3}$ be a disjoint union of $k+1+$ lines with $Y^{\prime \prime}=G_{\text {red }}$, and let $S$ be the support of the nilradical of $G$ and with $Q$ containing each tangent vector of a +lines of $G$; that is, we assume that $\operatorname{deg}(G \cap Q)=3(k+1)$ and that $\operatorname{Res}_{Q}(G)=Y^{\prime \prime}$. Let $E \subset Q$ be a general union of $z_{k}-1$ lines of type $(0,1)$. Set $X:=Y^{\prime} \cup G \cup E$. Deforming $E$ 
to a general union of $z_{k}-1$ lines of $\mathbb{P}^{3}$, we see that $X$ is in the closure of a set of elements of $L\left(3, a_{k}-1, k+1\right)_{\mathrm{Q}}$. Hence it is sufficient to prove that $h^{1}\left(\mathscr{I}_{X}(k)\right)=0$; that is, $h^{0}\left(\mathscr{I}_{X}(k)\right)=$ $(k+1) / 3$. Since $\operatorname{Res}_{Q}(X)=Y$ and $h^{1}\left(\mathscr{I}_{Y}(k-2)\right)=0$, it is sufficient to prove that $h^{1}\left(Q, \mathscr{I}_{X \cap Q}(k)\right)=0$; that is, $h^{1}\left(Q, \mathscr{I}_{\operatorname{Res}_{E}\left(\mathrm{Q} \cap\left(Y^{\prime} \cup G\right)\right.}\left(k, k+1-z_{k}\right)\right)=0$. Since $E \cap X=\emptyset$, we have $\operatorname{Res}_{E}\left(Q \cap\left(Y^{\prime} \cup G\right)\right)=Q \cap\left(Y^{\prime} \cup G\right)$. The scheme $Q \cap\left(Y^{\prime} \cup G\right)$ is a general union of $2 \operatorname{deg}\left(Y^{\prime}\right)$ points of $Q$ and $k+1$ tangent vectors of $Q$. Hence it is sufficient to prove that $h^{1}\left(Q, \mathscr{I}_{Z}\left(k, k+1-z_{k}\right)\right)=0$ for a general union $Z$ of $k+1$ tangent vectors of $Q$; this is very easy; in characteristic zero we may also quote two general results: [15] and [16, Lemma $1.4]$.

(b) Assume that $k \equiv 0(\bmod 3)$. Take $Y, S, G, X$ as in step (a). In this case we easily get $h^{i}\left(\mathscr{I}_{X}(k)\right)=0, i=0,1$.

(c) By Remark 3 to prove $D_{3 x+2}^{\prime \prime}$ for all $x \geq 0$, it is sufficient to prove $D_{3 x+2}^{\prime \prime}$ for all $x \geq 2$. As in step (a), we get $D_{3 x+2}^{\prime \prime}$.

(d) Assume that $k \equiv 1(\bmod 3)$. Take $Y \in L\left(3, a_{k-2}-\right.$ $(k-1) / 3,(k-1) / 3)_{\mathrm{Q}}$ satisfying $D_{k-2}^{\prime}$. For a general $Y$, we may assume that $\operatorname{Res}_{Q}(Y)=Y$ and that $Y \cap Q$ is a general union of $2 a_{k-2}$ points of $Q$. Write $Y=Y^{\prime} \sqcup Y^{\prime \prime}$ with $Y^{\prime \prime}$ union of the +lines. Fix a set $S \subset Y^{\prime} \cap Q$ such that $\sharp(S)=k+1-$ $(k-1) / 3$ and each line of $Y^{\prime}$ contains at most one point of $S$. Write $Y^{\prime}=Y_{1} \sqcup Y_{2}$ with $Y_{2}$ the union of the lines of $Y^{\prime \prime}$ containing a point of $S$. Let $G \subset \mathbb{P}^{3}$ be a disjoint union of $k+1-(k-1) / 3+$ lines with $Y_{2}$ as its support and $S$ as the support of its nilradical and with $Q$ containing each tangent vector of a +lines of $G$. Let $E \subset Q$ be a general union of $z_{k}-1$ lines of type $(0,1)$. Set $X:=Y_{1} \cup G \cup Y^{\prime \prime} \cup E$. As in step (a), we get $h^{i}\left(\mathscr{I}_{X}(k)\right)=0, i=0,1$, and $X$ is a flat limit of a family of elements of $L\left(3, a_{k}-1, k+1\right)_{\mathrm{Q}}$.

Assertion $C_{k}, k \geq 3$. Set $\epsilon:=0$ if $k \equiv 0,1(\bmod 3), \epsilon:=0$ if $k=5, \epsilon:=(k+1) / 3-1$ if $k \equiv 2(\bmod 6)$, and $\epsilon:(k+1) / 3-2$ if $k \equiv 5(\bmod 6)$ and $k \geq 11$. There are a union $F \subset Q$ of $\lceil y / 2\rceil$ different lines of type $(1,0), B \subset F, \sharp(B)=y$, and $\sharp(B \cap$ $L) \leq 2$ for each component $L$ of $F, Y \in L\left(3, a_{k}-1-y, y\right), B$ is the support of the nilpotent sheaf of $Y$, and $h^{1}\left(\mathscr{I}_{Y}(k)\right)=0$.

Remark 7. Take a general $B \subset \mathbb{P}^{3}$ with $\sharp(B) \leq 6 . B$ is a contained in the union $T$ of 3 disjoint lines and $T$ is contained in a smooth quadric with, say, $T$ of type $(3,0)$. Since a general element of $L\left(3, a_{k}-1-y, y\right)$ has maximal rank [10], to prove $C_{k}$, it is sufficient to check the existence of $Y$ for all $y>7$. In particular $C_{k}$ is true if $k \leq 6$.

Lemma 8. $C_{k}$ is true for all $k \geq 3$.

Proof. By Remark 7, we may assume that $k \geq y \geq 7$. In steps (a), (b), and (c), we assume that $y \geq z_{k}$, while in step (d), we handle the easier case $y \leq z_{k}-1$.

(a) Assume that $k \equiv 2(\bmod 3)$ and $y \geq z_{k}$. Take a general $Y \in L\left(3, a_{k-4}, 0\right)$. We have $h^{i}\left(\mathscr{I}_{Y}(k-4)\right)=0, i=0,1$. Let $E \subset$ $Q$ be a general union of $z_{k-2}$ lines of type $(0,1)$. Fix the union $F \subset Q$ of $\left\lfloor z_{k-2} / 2\right\rfloor$ general lines of type $(1,0)$. As in [8] (or as in the proof of Lemma 4), we get $h^{i}\left(\mathscr{I}_{Y \cup E}(k-2)\right)=0, i=0,1$. For each line $L \subset F$, we fix two of the lines of $E$ (and call them $E_{L}^{\prime}$ and $E_{L}^{\prime \prime}$ ) with the condition that $\cup_{L \subseteq F} E_{L}^{\prime} \cup E_{L}^{\prime \prime}$ is the union of $2\left\lfloor z_{k-2} / 2\right\rfloor$ distinct lines. Set $S_{1}:=\cup_{L \subseteq F} L \cap\left(E_{L}^{\prime} \cup E_{L}^{\prime \prime}\right)$. Write $E=E_{1} \sqcup E_{0}$ with $E_{0}=\emptyset$ if $z_{k-2}$ is even and $E_{0}$ the only line of $E$ not containing a point of $S_{1}$ if $z_{k-2}$ is odd. Equivalently, take $S_{1} \subset F \cap E$ such that $\sharp\left(S_{1} \cap L\right)=2$ for every line $L \subset F$ and $\sharp\left(S_{1} \cap L\right) \leq 1$ for every line $L \subset E$. There are a smooth and connected affine curve $\Delta, o \in \Delta$, a flat family $\left\{E_{\lambda}\right\}_{\lambda \in \Delta}$ of subschemes of $\mathbb{P}^{3}$ with $E_{\lambda} \in L\left(3, z_{k-2}, 0\right)$ for all $\lambda \in \Delta$, $E_{o}=E, E_{\lambda}$ transversal to $Q$ for all $\lambda \neq o$ and such that for each $L \in F$ and each $\lambda \in \Delta \backslash\{o\}$ exactly two of the lines of $E_{\lambda}$ meet $L$; that is, we deform the lines of $E_{L}^{\prime}$ and $E_{L}^{\prime \prime}$ to lines $E_{\lambda L}^{\prime}$, $E_{\lambda L}^{\prime \prime}$ transversal to $Q$, but meeting $L$. Notice that for a general $\lambda \in \Delta$, the point of $E_{\lambda L}^{\prime} \cap(Q \backslash L)$ and the point of $E_{\lambda L}^{\prime \prime} \cap(Q \backslash L)$ may be general points of $Q$. Hence we may find this flat family with the additional condition that for a general $\lambda \in \Delta$ the set $\left(Y \cup E_{\lambda}\right) \cap(Q \backslash F)$ is a general union of $2 a_{k-2}+2 z_{k-2}-2\left\lfloor z_{k-2} / 2\right\rfloor$ points of $Q$. Fix a general $\lambda \in \Delta$ and write $E_{\lambda}=E_{1 \lambda} \sqcup E_{0 \lambda}$ with $S_{1} \subset E_{1 \lambda}$ and $E_{0 \lambda}$ either empty (case $z_{k-2}$ even) or $E_{0 \lambda}$ a line (case $z_{k-2}$ odd). Let $G \subset Q$ be a general union of $z_{k}-1$ lines of type $(0,1)$. Fix a general union $F^{\prime} \subset Q$ of $\lceil y / 2\rceil-\left\lfloor z_{k-2} / 2\right\rfloor$ lines of type $(1,0)$ and any union $G_{2} \subset G$ of $y-2\left\lfloor z_{k-2} / 2\right\rfloor$ components of $G$. Write $G=G_{2} \sqcup G_{0}$. We take $S_{2} \subset F^{\prime} \cap G_{2}$ so that $\sharp\left(S_{2}\right)=y-2\left\lfloor z_{k-2} / 2\right\rfloor, \sharp\left(S_{2} \cap L\right) \leq 2$ for every line $L \subset F^{\prime}$ and $\sharp\left(S_{2} \cap L\right) \leq 1$ for every line $L \subset E^{\prime}$; to check that this is possible we need to check that $z_{k}-1 \geq y-2\left\lfloor z_{k-2} / 2\right\rfloor$ (call $\diamond$ this inequality). First assume that $k=6 t+2$. We have $z_{6 t+2}=4 t+2$ and $z_{6 t}=4 t+1$; we have $z_{6 t+1}-1+2\left\lfloor z_{6 t} / 2\right\rfloor=$ $4 t+1+4 t=k+(k+1) / 3-1$, and hence $\diamond$ is true by our definition of the integer $\epsilon$. Now assume that $k=6 t+5$; we have $z_{6 t+5}=4 t+4, z_{6 t+3}=4 t+3$, and hence $z_{6 t+5}-1+2\left\lfloor z_{6 t+3} / 2\right\rfloor=$ $4 t+3+4 t+2=k+(k+1) / 3-2$; hence $\diamond$ is true in this case. Write $E^{\prime}=E_{1}^{\prime} \sqcup E_{0}^{\prime}$ with $E_{0}^{\prime}$ the union of all lines of $E^{\prime}$ containing no point of $S_{2}$. Let $Y_{1}$ be a general union of +lines with $E_{1}$ as its support and $S_{1}$ as the support of the nilradical and the nilradical contained in $Q$, so that $\operatorname{Res}_{Q}\left(Y_{1}\right)=E_{1 \lambda}$ and $\operatorname{deg}\left(Y_{1} \cap Q\right)=3 \operatorname{deg}\left(Y_{1}\right)$. Let $Y_{2} \subset Q$ be a general union of +lines contained in $Q$, supported by $G_{2}$ and with $S_{2}$ as the support of the nilradical. Set $X:=Y \cup Y_{1} \cup Y_{2} \cup E_{0 \lambda} \cup G_{0}$. It is sufficient to prove that $h^{1}\left(\mathscr{I}_{X}(k)\right)=0$. Since $\operatorname{Res}_{Q}(X)=$ $Y \cup E_{\lambda}$, it is sufficient to prove that $h^{1}\left(Q, \mathscr{I}_{\mathrm{Q} \cap X}(k)\right)=0$. Since $\operatorname{Res}_{G}(Q \cap S)=Y \cap Q \cup\left(Y_{1} \cap Q\right) \cup S_{2}$ and $y \leq k+1+(k+1) / 3$, it is sufficient to prove that the union $W$ of $S_{2}$ and the degree two connected components of $Y_{1} \cap Q$ satisfies $h^{1}\left(Q, \mathscr{I}_{W}(k, k+1-\right.$ $\left.\left.z_{k}\right)\right)=0$. Write $W=W^{\prime} \sqcup S_{2}$. Since $F^{\prime}$ is general, $\sharp\left(S_{2} \cap L\right) \leq$ 2 for each line $L \subseteq F^{\prime}$, and $S_{2} \cap L$ is general in $L$ for each line $L \subseteq F^{\prime}$ and $k+1-z_{\mathrm{k}} \geq 2$, it is sufficient to prove that $h^{1}\left(Q, \mathscr{I}_{W^{\prime}}\left(k, k+1-z_{k}\right)\right)=0$. In characteristic zero this is true by either [15] or [16, Lemma 1.4]; in positive characteristic one could see that there are only $\sharp\left(S_{2}\right)$ tangent vectors and $k+1+$ $\left(k+1-z_{k}\right) \sim y-\left\lfloor z_{k-2} / 2\right\rfloor=\sharp\left(S_{2}\right)$.

(b) Assume that $k \equiv 0(\bmod 3)$ and $y \geq z_{k}$. Fix a general $Y \in L\left(3, a_{k-4}-(k-3) / 2,(k-3) / 2\right)_{\mathrm{Q}}$. By $D_{k-4}^{\prime}$, we have $h^{i}\left(\mathscr{I}_{Y}(k-4)\right)=0, i=0,1($ Lemma 4$)$. Let $E \subset Q$ be a general union of $z_{k-2}$ lines of type $(0,1)$, with the restriction that $(k-3) / 2$ of them contain one of the points of the support of the nilradical of $Y$. As in step (d) of the proof of Lemma 6, we get $h^{i}\left(\mathscr{I}_{Y}(k-2)\right)=0, i=0,1$. Let $F \subset Q$ be a general union of $\left\lfloor z_{k-2} / 2\right\rfloor$ lines of type $(1,0)$. Fix $S_{1} \subset F \cap E$ such that $\sharp\left(S_{1} \cap L\right)=2$ for every line $L \subset F$ (and hence 
$\left.\sharp\left(S_{1}\right)=2\left\lfloor z_{k-2} / 2\right\rfloor\right)$ and $\sharp\left(S_{1} \cap L\right) \leq 1$ for every line $L \subset E$. There are a smooth and connected affine curve $\Delta, o \in \Delta$, a flat family $\left\{E_{\lambda}\right\}_{\lambda \in \Delta}$ with $E_{\lambda} \in L\left(3, z_{k-2}, 0\right)$ for all $\lambda \in \Delta, E_{o}=E, E_{\lambda}$ transversal to $Q$ for all $\lambda \neq o$ and that for each $L \in F$ and each $\lambda \in \Delta \backslash\{o\}$ exactly two of the lines of $E_{\lambda}$ meet $L$. As in step (a) for a general $\lambda$, the set $\left(Y \cup E_{\lambda}\right) \cap(Y \backslash F)$ is a general union of $2 a_{k-2}+2 z_{k-2}-2\left\lfloor z_{k-2} / 2\right\rfloor$ points of $Q$. Fix a general $\lambda \in \Delta$ and write $E_{\lambda}=E_{1 \lambda} \sqcup E_{0 \lambda}$ with $S_{1} \subset E_{1 \lambda}$ and $E_{0 \lambda}$ either empty (case $z_{k-2}$ even) or a line (case $z_{k-2}$ odd). Let $G \subset Q$ be a general union of $z_{k}-1$ lines of type $(0,1)$. Fix a general union $F^{\prime} \subset Q$ of $\lceil y / 2\rceil-\left\lfloor z_{k-2} / 2\right\rfloor$ lines of type $(1,0)$ and any union $G_{2} \subset G$ or $y-2\left\lfloor z_{k-2} / 2\right\rfloor$ components of $G$. Write $G=G_{2} \sqcup G_{0}$ with $G_{0}$ the union of all lines of $G$ not intersecting $S_{2}$. We take $S_{2} \subset F^{\prime} \cap G^{\prime}$ so that $\sharp\left(S_{2}\right)=y-2\left\lfloor z_{k-2} / 2\right\rfloor, \sharp\left(S_{2} \cap L\right) \leq 2$ for every line $L \subset F^{\prime}$, and $\sharp\left(S_{2} \cap L\right) \leq 1$ for every line $L \subset E^{\prime}$; to check that this is possible we need to check that $z_{k}-1 \geq y-2\left\lfloor z_{k-2} / 2\right\rfloor$ and this is true and easier than in step (a), because $\epsilon=0$. Write $E^{\prime}=E_{1}^{\prime} \sqcup E_{0}^{\prime}$ with $E_{0}^{\prime}$ the union of all lines of $E^{\prime}$ containing no point of $S_{1}$. Let $Y_{1}$ be a general union of +lines with $E_{1}$ as its support and $S_{1}$ as the support of the nilradical and the nilradical contained in $Q$, so that $\operatorname{Res}_{Q}\left(Y_{1}\right)=E_{1 \lambda}$ and $\operatorname{deg}\left(Y_{1} \cap Q\right)=3 \operatorname{deg}\left(Y_{1}\right)$. Let $Y_{2} \subset Q$ be a general union of +lines contained in $Q$, supported by $G_{2}$ and with $S_{2}$ as the support of the nilradical. Set $X:=Y \cup Y_{1} \cup Y_{2} \cup E_{0 \lambda} \cup G_{0}$. It is sufficient to prove that $h^{1}\left(\mathscr{I}_{X}(k)\right)=0$. Since $\operatorname{Res}_{\mathrm{Q}}(X)=$ $Y \cup E_{\lambda}$, it is sufficient to prove that $h^{1}\left(Q, \mathscr{I}_{\mathrm{Q} \cap X}(k)\right)=0$. Since $\operatorname{Res}_{G}(Q \cap S)=Y \cap Q \cup\left(Y_{1} \cap Q\right) \cup S_{2}$ and $y \leq k+1$, it is sufficient to prove that the union $W$ of $S_{2}$ and the degree two connected components of $Y_{1} \cap Q$ satisfies $h^{1}\left(Q, \mathscr{I}_{W}\left(k, k+1-z_{k}\right)\right)=0$. Write $W=W^{\prime} \sqcup S_{2}$. Since $F^{\prime}$ is general, $\sharp\left(S_{2} \cap L\right) \leq 2$ for each line $L \subseteq F^{\prime}$, and $S_{2} \cap L$ is general in $L$ for each line $L \subseteq F^{\prime}$ and $k+1-z_{k} \geq 2$, it is sufficient to prove that $h^{1}\left(Q, \mathscr{I}_{W^{\prime}}\left(k, k+1-z_{k}\right)\right)=0$. In characteristic zero this is true by either [15] or [16, Lemma 1.4]; in positive characteristic one could see that there are only $2\left\lfloor z_{k-2} / 2\right\rfloor$ tangent vectors and $k+1+k+1-z_{k} \sim 2\left\lfloor z_{k-2} / 2\right\rfloor$.

(c) Assume that $k \equiv 1(\bmod 3)$ and $y \geq z_{k}$. Fix a general $Y \in L\left(3, a_{k-4}, 0\right)$. We have $h^{i}\left(\mathscr{I}_{Y}(k-4)\right)=0, i=0$, 1 . Let $E \subset Q$ be a general union of $z_{k-2}$ lines of type $(0,1)$. Let $F \subset Q$ be a general union of $\left\lfloor z_{k-2} / 2\right\rfloor$ lines. Fix $S_{1} \subset F \cap E$ such that $\sharp\left(S_{1} \cap L\right)=2$ for every line $L \subset F$ and $\sharp\left(S_{1} \cap L\right) \leq 1$ for every line $L \subset E$. There are a smooth and connected affine curve $\Delta, o \in \Delta$, a flat family $\left\{E_{\lambda}\right\}_{\lambda \in \Delta}$ with $E_{\lambda} \in L\left(3, z_{k-2}, 0\right)$ for all $\lambda \in \Delta, E_{o}=E, E_{\lambda}$ transversal to $Q$ for all $\lambda \neq o$ and that for each $L \in F$ and each $\lambda \in \Delta \backslash\{0\}$ exactly two of the lines of $E_{\lambda}$ meet $L$. As in step (a) for a general $\lambda$, the set $\left(Y \cup E_{\lambda}\right) \cap(Y \backslash F)$ is a general union of $2 a_{k-2}+2 z_{k-2}-2\left\lfloor z_{k-2} / 2\right\rfloor$ points of $Q$. In this flat family each $E_{\lambda}, \lambda \neq 0$, is a disjoint union of lines, and only $E_{o}$ has sundials as some of its connected components. Fix a general $\lambda \in \Delta$ and write $E_{\lambda}=E_{1 \lambda} \sqcup E_{0 \lambda}$ with $S_{1} \subset E_{1 \lambda}$ and $E_{0 \lambda}$ either empty (case $z_{k-2}$ even) or a line (case $z_{k-2}$ odd). Fix $\gamma \subset E_{1} \backslash E_{1} \cap F$ such that $\sharp(\gamma)=b_{k-2}=(k-1) / 3$. For each line $R$ of $E$ and each $\mu \in \Delta$ call $R_{\mu}$, the line of $E_{\mu}$ such that $\left\{R_{\mu}\right\}$ is an algebraic family of lines. Let $\gamma_{\lambda}$ be the intersection with $Q \backslash F$ of the subcurve $E_{\lambda}(\gamma)$ of $E_{\lambda}$ corresponding to the lines $R_{\lambda}$ with $R \cap \gamma \neq \emptyset$. The set $\gamma_{\lambda}$ is a general subset of $Q$ with cardinality $b_{k-2}$. Let $\widetilde{E}_{\lambda}$ be the union of $E_{\lambda} \backslash E_{\lambda}(\gamma)$ and $b_{k-2}$ general +lines contained in $Q$, with $E_{\lambda}(\gamma)$ as their support and with $\gamma_{\lambda}$ as the support of their nilradicals; for each +line $J$ of $\widetilde{E}$, we assume that the tangent vector $v$ of $J$ corresponding to the nilpotent sheaf of $J$ is not tangent to $Q$; that is, we assume that the scheme $J \cap Q$ has degree two and it is the disjoint union of two points; with these restrictions we have $\operatorname{Res}_{Q}\left(\widetilde{E}_{\lambda}\right)=\widetilde{E}_{\lambda}$. Let $G \subset Q$ be a general union of $z_{k}-1$ lines of type $(0,1)$ with the only restriction that $b_{k-2}$ of them contain a point of $\gamma_{\lambda}$. Fix a general union $F^{\prime} \subset Q$ of $\lceil y / 2\rceil-\left\lfloor z_{k-2} / 2\right\rfloor$ lines of type $(1,0)$ and any union $G_{2} \subset G$ or $y-2\left\lfloor\left(a_{k-2}-a_{k-4}\right) / 2\right\rfloor$ components of $G$. Write $G=G_{2} \sqcup G_{0}$ with $G_{0}$ the union of all lines of $G$ not intersecting $S_{2}$. We take $S_{2} \subset F^{\prime} \cap G^{\prime}$ so that $\sharp\left(S_{2}\right)=y-\left\lfloor z_{k-2} / 2\right\rfloor, \sharp\left(S_{2} \cap L\right) \leq 2$ for every line $L \subset$ $F^{\prime}$ and $\sharp\left(S_{2} \cap L\right) \leq 1$ for every line $L \subset E^{\prime}$; to check that this is possible we need to check that $z_{k}-1 \geq y-2\left\lfloor z_{k-2} / 2\right\rfloor$ and this is true and easier than in step (a), because $\epsilon=0$. Write $E^{\prime}=E_{1}^{\prime} \sqcup E_{0}^{\prime}$ with $E_{0}^{\prime}$ the union of all lines of $E^{\prime}$ not containing a point of $S_{2}$. Let $Y_{1}$ be a general union of +lines with $E_{1}$ as its support and $S_{1}$ as the support of the nilradical and the nilradical contained in $Q$, so that $\operatorname{Res}_{Q}\left(Y_{1}\right)=E_{1 \lambda}$ and $\operatorname{deg}\left(Y_{1} \cap Q\right)=3 \operatorname{deg}\left(Y_{1}\right)$. Let $Y_{2} \subset Q$ be a general union of +lines contained in $Q$, supported by $G_{2}$ and with $S_{2}$ as the support of the nilradical. Set $X:=Y \cup Y_{1} \cup Y_{2} \cup \widetilde{E}_{0 \lambda} \cup G_{0}$. It is sufficient to prove that $h^{1}\left(\mathscr{I}_{X}(k)\right)=0$. Since $\operatorname{Res}_{Q}(X)=$ $Y \cup E_{\lambda}$, it is sufficient to prove that $h^{1}\left(Q, \mathscr{I}_{\mathrm{Q} \cap X}(k)\right)=0$. Since $\operatorname{Res}_{G}(Q \cap S)=Y \cap Q \cup\left(Y_{1} \cap Q\right) \cup S_{2}$ and $y \leq k+1+(k+1) / 3$, it is sufficient to prove that the union $W$ of $S_{2}$ and the degree two connected components of $Y_{1} \cap Q$ satisfies $h^{1}\left(Q, \mathscr{I}_{W}(k, k+1-\right.$ $\left.\left.z_{k}\right)\right)=0$. Write $W=W^{\prime} \sqcup S_{2}$. Since $F^{\prime}$ is general, $\sharp\left(S_{2} \cap L\right) \leq$ 2 for each line $L \subseteq G$, and $S_{2} \cap L$ is general in $L$ for each line $L \subseteq G$ and $k+1-z_{k} \geq 2$, it is sufficient to prove that $h^{1}\left(Q, \mathscr{I}_{W^{\prime}}\left(k, k+1-z_{k}\right)\right)=0$. In characteristic zero this is true by either [15] or [16, Lemma 1.4]; in positive characteristic one could see that there are only $2\left\lfloor z_{k-2} / 2\right\rfloor$ tangent vectors and $k+1+k+1-z_{k} \sim 2\left\lfloor z_{k-2} / 2\right\rfloor$.

(d) From now on we assume that $y \leq z_{k}-1$.

(d1) Assume that $k \equiv 2(\bmod 3)$. Fix a general $Y \in$ $L\left(3, a_{k-2}, 0\right)$. We have $h^{i}\left(\mathscr{I}_{Y}(k-2)\right)=0, i=0,1$ [8]. Fix a general union $F \subset Q$ of $\lceil y / 2\rceil$ lines of type $(1,0)$ and a general union $E \subset Q$ of $z_{k}-1$ lines of type $(0,1)$. Write $E=E_{1} \sqcup E_{0}$ with $\operatorname{deg}\left(E_{1}\right)=y$. Fix $S \subset E_{1} \cap F$ such that $\sharp(S)=y, \sharp(S \cap L) \leq$ 2 for all lines $L \subseteq F$ and $\sharp(S \cap R)=1$ for each line $R \subseteq E_{1}$. Let $G \subset Q$ be a general union of $y$ +lines with $E_{1}$ as their support and $S$ as the support of their nilradical. Set $X:=Y \cup G \cup E_{0}$; it works, because $h^{1}\left(Q, \mathscr{I}_{X \cap Q}(k)\right)=0$ since $y \leq k+1+(k+1) / 3$, $\operatorname{deg}(E)=z_{k}-1$, and $h^{0}\left(\mathcal{O}_{\mathrm{Qn} X}(k)\right) \leq(k+1)^{2}$.

(d2) Assume that $k \equiv 0(\bmod 3)$. Fix a general $Y \in$ $L\left(3, a_{k-2}, 0\right)$. We have $h^{i}\left(\mathscr{I}_{Y}(k-2)\right)=0, i=0,1$. Fix a general union $F \subset Q$ of $\lceil y / 2\rceil$ lines of type $(1,0)$ and a general union $E \subset Q$ of $z_{k}-1$ lines of type $(0,1)$. Write $E=E_{1} \sqcup E_{0}$ with $\operatorname{deg}\left(E_{1}\right)=y$. Fix $S \subset E_{1} \cap F$ such that $\sharp(S)=y, \sharp(S \cap L) \leq 2$ for all lines $L \subseteq F$ and $\sharp(S \cap R)=1$ for each line $R \subseteq E_{1}$. Let $G \subset Q$ be a general union of $y+$ lines with $E_{1}$ as their support and $S$ as the support of their nilradical. Set $X:=Y \cup G \cup E_{0}$; it works, because $h^{1}\left(Q, \mathscr{I}_{X \cap Q}(k)\right)=0$ since $y \leq k+1, \operatorname{deg}(E)=z_{k}-1$, and $h^{0}\left(\mathcal{O}_{\mathrm{Q} \cap X}(k)\right) \leq(k+1)^{2}$.

(d3) Assume that $k \equiv 1(\bmod 3)$. We have $b_{k-2}=(k-$ 1)/3. Fix a general $Y \in L\left(3, a_{k-2}-(k-1) / 3,(k-1) / 3\right)_{Q}$. 
We have $h^{i}\left(\mathscr{I}_{Y}(k-2)\right)=0, i=0,1$ by $D_{k-2}^{\prime}($ Lemma 6$)$. Fix a general union $F \subset Q$ of $\lceil y / 2\rceil$ lines of type $(1,0)$ and a general union $E \subset Q$ of $z_{k}-1$ lines of type $(0,1)$ with the only restriction that $(k-1) / 3$ of them contain a point of the support of the nilradical of $Y$. Write $E=E_{1} \sqcup E_{0}$ with $\operatorname{deg}\left(E_{1}\right)=y$. Fix $S \subset E_{1} \cap F$ such that $\sharp(S)=y, \sharp(S \cap L) \leq 2$ for all lines $L \subseteq F$ and $\sharp(S \cap R)=1$ for each line $R \subseteq E_{1}$. Let $G \subset Q$ be a general union of $y+$ lines with $E_{1}$ as their support and $S$ as the support of their nilradical. Set $X:=Y \cup G \cup E_{0}$; it works, because $h^{1}\left(Q, \mathscr{I}_{X \cap Q}(k)\right)=0$ since $y \leq k+1, \operatorname{deg}(E)=z_{k}-1$, and $h^{0}\left(\mathcal{O}_{\mathrm{Q} \cap X}(k)\right) \leq(k+1)^{2}$.

We also need the following elementary and well-known lemma (see, e.g., [6, Lemma 2]).

Lemma 9. Fix integer $k \geq 2, y>0$, a closed scheme $X \subset$ $\mathbb{P}^{3}$ such that $h^{1}\left(\mathscr{I}_{X}(k)\right)=0$ and a general set $S \subset Q$ such that $\sharp(S)=y$. If $y \leq h^{0}\left(\mathscr{I}_{X}(k)\right)-h^{0}\left(\mathscr{I}_{\operatorname{Res}_{Q}(X)}(k-2)\right)$, then $h^{1}\left(\mathscr{I}_{X \cup S}(k)\right)=0 \operatorname{If}^{0}\left(\mathscr{I}_{\operatorname{Res}_{Q}}(k-2)\right)=0$ and $y \geq h^{0}\left(\mathscr{I}_{X}(k)\right)$, then $h^{0}\left(\mathscr{I}_{X \cup S}(k)\right)=0$.

\section{Proof of Theorem 1}

In this section we prove Theorem 1 . We may assume that $a>0$ (by [8]) and $t>0$ (by the Alexander-Hirschowitz theorem). Set $\delta:=\left(\begin{array}{c}k+3 \\ 3\end{array}\right)-(k+1) t-4 a$. By increasing or decreasing $a$, we reduce to the case $-3 \leq \delta \leq 3$. Since any 2 -point contains a point if $a>0$ and Theorem 1 is true for the triple $(t, a-1, k)$ with $\delta=-1$ (i.e., if $h^{0}\left(\mathscr{I}_{X}(k)\right)=1$ for a general $X \in Z(3, t, a-$ $1)$, then it is true for the triple $(t, a, k)$ with $\delta=4)$. Hence it is sufficient to check all triples $(t, a, k)$ with $-3 \leq \delta \leq 2$. In characteristic zero we may avoid all cases with $\delta=2$ quoting either [15] or [16, Lemma 1.4]. The cases $k=3,4,5$ are true by $[6$, Propositions $1,2,3]$. In steps (a) and (b), we assume that $k \geq 9$ and that Theorem 1 is true in degree $k-2$ and $k-4$. See steps (c), (d), and (e) for the cases $k=6,7,8$.

(a) Assume that $a \geq(4 k+2) / 3$. If $3 a+2 t \geq(k+1)^{2}+4$, then set $g:=0$. If $3 a+2 t \leq(k+1)^{2}+3$, then let $g$ be the minimal positive integer such that $3 a+2 t+g(k-1) \geq(k+1)^{2}+4$.

Claim 1. We have $g \leq k-1$.

Proof of Claim 1. Assume that $g \geq k$. We get $3 a+2 t+(k-1)^{2} \leq$ $(k+1)^{2}+3$; that is, $3 a+2 t \leq 4 k+3$. Since $t>0$, we get $3 a \leq 4 k+1$, a contradiction.

Claim 2. We have $g<t$.

Proof of Claim 2. Assume that $g \leq t$. The minimality of the integer $g$ implies $3 a+2 t+(t-1)(k-1) \leq(k+1)^{2}+4$; that is, $3 a+(k+1) t \leq k^{2}+3 k+4$, contradicting the inequality $\delta \geq-3$.

Set $\left.w:=\left\lfloor(k+1)^{2}-2 t-(k-1) g\right) / 3\right\rfloor$ and $z:=(k+1)^{2}-$ $2 t-(k-1) g-3 w$. We have $0 \leq z \leq 2$ and $3 w+z+2(t-g)=$ $(k+1)(k+1-g)$. Since $z \leq 2$ and $3 a+2 t+g(k-1) \geq$ $(k+1)^{2}+4$, we get $a \geq w+z$. Let $Y$ be a general element of $Z(3, t-g, a-w-z)$. Fix a general $S \cup S^{\prime} \subset Q$ such that $\sharp(S)=w$,
$\sharp\left(S^{\prime}\right)=z$, and $S \cap S^{\prime}=\emptyset$. Let $E \subset Q$ be a general union of $g$ lines of type $(0,1)$. Since $g<t$, we have $3 w<(k+1)(k+1-g)$. Since $k-g>0$, we have $h^{1}\left(Q, \mathscr{I}_{2 S \cap Q}(k, k-g)\right)=0[17,18]$ and [19, Corollary 4.6] (the latter one only if $k-g \geq 2$, but the case $k-g=1$ is elementary, because $3 w<k+1)$. Since $Q \cap Y$ is a general subset with cardinality $2 t-2 g$ and $3 w+z+2 t=(k+1)^{2}$, we get $h^{i}\left(\mathscr{I}_{(Y \cup 2 S) \cap Q \cup S^{\prime} \cup E}(k)\right)=0, i=$ 0,1 . By the Differential Horace Lemma for double points [1] and [5, Lemma 5] to prove that $h^{1}\left(\mathscr{I}_{X^{\prime}}(k)\right)=\max \{0, \delta\}$ for a general union $X^{\prime}$ of $Y \cup 2 S \cup E$ and $z$ general 2-points (and hence to prove Theorem 1 in this case), it is sufficient to prove that $h^{1}\left(\mathscr{I}_{Y \cup S \cup\left(S^{\prime} \cap Q\right)}(k-2)\right)=\max \{0, \delta\}$. We have $h^{0}\left(\mathcal{O}_{Y \cup S \cup\left(S^{\prime} \cap Q\right)}(k-2)\right)=\left(\begin{array}{c}k+1 \\ 3\end{array}\right)+\delta+2 z-2 w$. Assume that for the moment $\delta \leq 0$. Since $z \leq w$, we have $h^{0}\left(\mathcal{O}_{Y \cup S \cup\left(S^{\prime} \cap Q\right)}(k-2)\right) \leq$ $\left(\begin{array}{c}k+1 \\ 3\end{array}\right)$. Since $w \geq z$ and $\operatorname{deg}\left(2 S^{\prime}\right)-\operatorname{deg}\left(2 S^{\prime} \cap Q\right)=z$, we have $h^{0}\left(\mathcal{O}_{Y \cup 2 S^{\prime}}(k-2)\right) \leq\left(\begin{array}{c}k+1 \\ 3\end{array}\right)$. Since $\sharp\left(S^{\prime}\right)=z \leq 2$ and any two points of $\mathbb{P}^{3}$ are contained in a smooth quadric surface, $Y \cup 2 S^{\prime}$ may be considered as a general element of $Z(3, t-g, a-u)$. Since $h^{0}\left(\mathcal{O}_{Y \cup 2 S^{\prime}}(k-2)\right) \leq\left(\begin{array}{c}k+1 \\ 3\end{array}\right)$, the inductive assumption gives $h^{1}\left(\mathscr{I}_{Y \cup 2 S^{\prime}}(k-2)\right)=0$. Hence $h^{1}\left(\mathscr{I}_{Y \cup\left(S^{\prime} \cap Q\right)}(k-2)\right)=$ 0 . By Lemma 9 to prove that $h^{1}\left(\mathscr{I}_{Y \cup S \cup\left(S^{\prime} \cap Q\right)}(k-2)\right)$, it is sufficient to prove that $h^{0}\left(\mathscr{I}_{Y}(k-4)\right) \leq h^{0}\left(\mathscr{I}_{Y}(k-2)\right)-w-2 z$. Since $h^{1}\left(\mathscr{I}_{Y}(k-2)\right) \leq h^{1}\left(\mathscr{I}_{Y \cup 2 S^{\prime}}(k-2)\right)=0$, we have $h^{0}\left(\mathscr{I}_{Y}(k-2)\right)=w+2 z-\delta \geq w+2 z$. Hence it is sufficient to prove that $h^{0}\left(\mathscr{I}_{Y}(k-4)\right)$. The inductive assumption in degree $k-4$ shows that it is sufficient to prove that $h^{0}\left(\mathcal{O}_{Y}(k-4)\right) \geq$ $\left(\begin{array}{c}k-1 \\ 3\end{array}\right)$. We have $h^{0}\left(\mathcal{O}_{Y}(k-4)\right)=h^{0}\left(\mathcal{O}_{Y}(k-2)\right)-2(t-g)=$ $\left(\begin{array}{c}k+1 \\ 3\end{array}\right)+\delta-w-2 z-2(t-g)$. Since $3 w+z+2(t-g)=(k+1)^{2}$ and $\left(\begin{array}{c}k+1 \\ 3\end{array}\right)-\left(\begin{array}{c}k-1 \\ 3\end{array}\right)=(k-1)^{2}$, it is sufficient to check that $2 w \geq z-\delta+4 k$. Since $z \leq 2$ and $-\delta \leq 3$, it is sufficient to prove that $w \geq 2 k+3$. Assume that $w \leq 2 k+2$. Hence $3 w+z \leq 6 k+8$. Since $h^{0}\left(\mathscr{I}_{Y}(t-2)\right)>0$, we have $(k-1)(t-g) \leq\left(\begin{array}{c}k+1 \\ 3\end{array}\right)$; that is, $t-g \leq k(k+1) / 6$. Hence $3 w+z+2(t-g) \leq 6 k+8+k(k+1) / 3$, contradicting the inequality $\delta \geq-3$ for all $k \geq 6$.

Now assume that $\delta=1,2$. If $\delta \geq z$, then we just saw that $h^{0}\left(\mathscr{I}_{Y \cup S}(k-2)\right)=0$. Hence we may assume $z=2$ and $\delta=1$. Take a general $P \in Q$ and set $S_{1}:=S \cup\{P\}$. We have $h^{0}\left(Q, \mathscr{I}_{2 S_{1} \cap Q}(k)\right)=0$. Hence it is sufficient to prove that $h^{0}\left(\mathscr{I}_{Y \cup S_{1}}(k-2)\right)=0$. Since we checked that $h^{0}\left(\mathscr{I}_{A}(k-4)\right)=0$ (easier for $\delta>0)$ and that $h^{1}\left(\mathscr{I}_{A}(k-2)\right)=0$ (i.e., $h^{0}\left(\mathscr{I}_{A}(k-\right.$ $\left.2))=\sharp\left(S_{1}\right)\right)$, Lemma 9 gives $h^{i}\left(\mathscr{I}_{A \cup S_{1}}(k-2)\right)=0, i=0,1$.

(b) In this step we assume that $a<(4 k+2) / 3$. Define the integers $f$ and $f^{\prime}$ by the relations

$$
3 a+2 t+(k-1) f-f^{\prime}=(k+1)^{2}, \quad 0 \leq f^{\prime} \leq k-2 .
$$

Since $a>0$, we have $f \leq k+2$.

Claim 3. We have $f \leq k-1$ for all $k \geq 7$ and $f \leq k-2$ for all $k \geq 9$.

Proof of Claim 3. Assume that $f \geq k$. We get $3 a+2 t+(k-$ $1)^{2}<(k+1)^{2}$; that is, $3 a+2 t \leq 4 k+1$. For all $k \geq 7$, we get $4 a+(k+1) t \leq(k+1)(4 k+1) / 2<-3+\left(\begin{array}{c}k+3 \\ 3\end{array}\right)$, a contradiction. Now assume that $f=k-1$. We get $3 a+2 t \leq 5 k-2$. For all $k \geq 9$, we get $4 a+(k+1) t \leq(k+1)(5 k-2) / 2<-3+\left(\begin{array}{c}k+3 \\ 3\end{array}\right)$, a contradiction. 
Claim 4. We have $t-f \geq k-2$ for all $k \geq 7$.

Proof of Claim 4. Assume that $t \leq f+k-1$. Since $f \leq k-1$ by Claim 3 , we get $t \leq 2 k$. Hence $4 a+(k+1) t \leq 4(4 k+1) / 3+$ $2 k(k+1)<-3+\left(\begin{array}{c}k+3 \\ 3\end{array}\right)$, a contradiction for all $k \geq 7$.

Claim 5. We have $2 f \geq f^{\prime}$ for all $k \geq 4$.

Proof of Claim 5. Assume that $f \leq\left(f^{\prime}-1\right) / 2$. We get $3 a+$ $(k-1)\left(f^{\prime}-1\right) / 2-f^{\prime} \geq(k+1)^{2}$; that is, $3 a+(k-3) f^{\prime} / 2 \geq$ $k^{2}+3 k / 2+3 / 2$. Since $f^{\prime} \leq k-2$ and $3 a \leq 4 k+1$, we get a contradiction.

Claim 6. Either $t-f<a_{k-2}$ or $t-f=a_{k-2}, k \equiv 1(\bmod 3)$ and $a<(k-1) / 3$, or Theorem 1 holds for the pair $(t, a)$.

Proof of Claim 6. First assume that $t-f \geq a_{k-2}+1$. Fix a general $U \in L(3, t-f, 0)$. By [8], we have $h^{0}\left(\mathscr{I}_{U}(k-2)\right)=0$ and $h^{1}\left(\mathscr{I}_{U}(k-2)\right)=k-1-b_{k-2} \geq 2(k-1) / 3$. Let $E \subset Q$ be a general union of $f$ lines. Fix a general $S \subset Q$ such that $\sharp(S)=$ a. Since $h^{0}\left(Q, \mathscr{I}_{\mathrm{Q}(Y \cup 2 S) \cup E}(k)\right)=0$, Castelnuovo's sequence

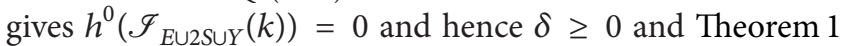
is true for the pairs $(t, a)$. The same proof works if $t-f=$ $a_{k-2}$ and either $b_{k-2}=0$ (i.e., $\left.k-2 \equiv 0,1(\bmod 3)\right)$ or $k-$ $2 \equiv 2(\bmod 3)$ and $a \geq(k-1) / 3($ in the latter case we quote Lemma 9 to get $h^{0}\left(\mathscr{I}_{\text {Y } \cup S}(k-2)\right)=0$.

(b1) Assume for the moment that either $k \equiv 0,2(\bmod 3)$, $t-f \neq a_{k-2}$, or $a \geq(k-1) / 3$. Let $S \subset Q$ be a general set with $\sharp(S)=a$. Let $E \subset Q$ be a general union of $f$ lines of type $(0,1)$. Claim 4 implies $t \geq f+f^{\prime}$. By Claim 6, we may assume that $f^{\prime} \leq 2 f$. Since $f^{\prime} \leq 2 f, f^{\prime} \leq k-2, t-f-f^{\prime} \geq 0$, and $t-f<$ $a_{k-2}$, Lemma 6 shows the existence $Y \in L\left(3, t-f-f^{\prime}, f^{\prime}\right)$ such that $h^{1}\left(\mathscr{I}_{Y}(k-2)\right)=0$, the nilradical of $\mathscr{O}_{Y}$ is supported by points of $E$, each scheme $Y \cap Q$ is reduced, and it is the union of $2(t-f)$ points, $f^{\prime}$ on $E$, and the remaining ones forming a general subset of $Q$ with cardinality $2(t-f)-f^{\prime}>0$. Since $k-f \geq 1$ and $2(t-f)-f^{\prime}>0$, we have $h^{1}\left(Q, \mathscr{I}_{2 S \cap Q}(k, k-\right.$ $f))=0$ [17]. Hence $h^{i}\left(Q, \mathscr{I}_{(Y \cup 2 S) \cap Q \cup E}(k)\right)=0, i=0,1$.

(b2) Now assume that $k \equiv 1(\bmod 3), a_{k-2}=t-f$, and $a<(k-1) / 3$. We modify step (b1) in the following way. Let $W$ be a general element of $L\left(3, a_{k-2}-(k-3) / 3,(k-3) / 3\right)_{Q}$. Since $D_{k-2}^{\prime}$ is true (Lemma 4), we have $h^{i}\left(\mathscr{I}_{W}(k-2)\right)=0, i=0,1$. Take any $Y \subseteq W$ with $Y \in L\left(3, t-f-f^{\prime}, f^{\prime}\right)_{\mathrm{Q}}$. We have $h^{1}\left(\mathscr{I}_{Y}(k-2)\right)=0$, because for each + line $T$ and each integer $t \geq 1$, the restriction map $\mathrm{H}^{0}\left(T, \mathcal{O}_{T}(t)\right) \rightarrow H^{0}\left(T_{\text {red }}, \mathcal{O}_{T_{\text {red }}}(t)\right)$ is surjective. Since $(t-3)\left(a_{k-2}-(k-1) / 3\right) \geq\left(\begin{array}{c}k+1 \\ 3\end{array}\right)$, we have $h^{0}\left(\mathscr{I}_{Y}(k-4)\right)=0$. Hence Lemma 9 gives that either $h^{0}\left(\mathscr{I}_{Y \cup S}(k-2)\right)=0$ or $h^{1}\left(\mathscr{I}_{Y \cup S}(k-2)\right)=0$. Since $3 a+2(t-$ $f)+(k+1) f-f^{\prime}=(k+1)^{2}$, we have $f \leq z_{k} \leq k-2$. Hence we may apply $[17,18]$ or $[19$, Corollary 4.6].

(c) Assume that $k=6$. Since $\left(\begin{array}{l}9 \\ 3\end{array}\right)=84$, it is sufficient to consider the following triples $(t, a, \delta):(1,19,-1)$, $(1,20,3),(2,17,-2),(2,18,2),(3,15,-3),(3,16,1),(4,14,0)$, $(5,12,-1),(5,13,3),(6,10,-2),(6,11,2),(7,8,-3),(7,9,1)$, $(8,7,0),(9,5,-1),(9,6,3),(10,3,-2),(10,4,2),(11,1,-3)$, and $(11,2,1)$. In all cases we take inside $Q$ a general union of $f$ lines of type $(0,1)$ and $S \cup S^{\prime}$ with $\sharp(S)=u, \sharp\left(S^{\prime}\right)=v$, $3 u+v+2(t-f)=8(8-f)$, and $0 \leq v \leq 2$. Outside $Q$, we have a general $Y \in Z(3, t-f, a-u-v)$.

(c1) Assume that $1 \leq t \leq 3$. Take $f=t$. The triples $(t, u, v)$ are the following ones: $(1,18,2),(2,16,0)$, and $(3,13,1)$. Hence we need to modify the proof in the case $(t, a, \delta)=$ $(1,19,-1)$. In that case we take $S^{\prime}$ with $\sharp\left(S^{\prime}\right)=1$; that is, take $v=1$. With this modification, we get $a \geq u+v$ in all cases. To use the Alexander-Hirschowitz theorem in degree 4, we need to check that $a-u<9$. We need to check that $h^{0}\left(\mathscr{I}_{Y}(2)\right) \leq$ $h^{0}\left(\mathscr{I}_{Y}(4)\right)-u-2 v$. In all cases we have $a-u-v \leq 2$, and hence $h^{1}\left(\mathscr{I}_{Y}(1)\right) \leq 1$. Hence $h^{0}\left(\mathscr{I}_{Y}(4)\right)-h^{0}\left(\mathscr{I}_{Y}(2)\right) \geq 5^{2}-1$.

(c2) Assume that $t=4$. Take $f=3$, and hence $(u, v)=$ $(12,2)$. Hence $Y$ is a line, $h^{1}\left(\mathscr{I}_{Y \cup 2 S^{\prime}}(4)\right)=0$ and $h^{0}\left(\mathscr{I}_{Y}(4)\right)-$ $h^{0}\left(\mathscr{I}_{Y}(2)\right)=25 \geq u+2 v$.

(c3) Assume that $5 \leq t \leq 10$. Take $f=4$. Since $t-f>$ 0 and $Y \cap Q$ is a general union of $2(t-f)$ points, we have $h^{1}\left(Q, \mathscr{I}_{2 S \cap Q}(6,2)\right)=0$. We have $u=\lfloor(29-2 t) / 3\rfloor$ and $v=$ $29-2 t-3 u$. Hence the triples $(t, u, v)$ are the following ones: $(5,6,1),(6,5,2),(7,5,0),(8,4,1),(9,3,2)$, and $(10,3,0)$. We have $u \geq v, u \geq v+\delta, a \geq u+v, h^{1}\left(\mathscr{I}_{Y \cup 2 S^{\prime}}(4)\right)=0[6$, Proposition 2], and $h^{0}\left(\mathscr{I}_{Y}(2)\right)=0$.

(c4) Assume that $t=11$. If $\delta=-3$, then we take $f=5$ and $S=S^{\prime}=\emptyset$. It is sufficient to use that $h^{1}\left(\mathscr{I}_{W}(4)\right)=0$ for a general $W \in Z(3,6,1)[6$, Proposition 2].

(d) Assume that $k=7$. Since $\left(\begin{array}{c}10 \\ 3\end{array}\right)=120$ and 120 is divided both by 8 and by 4 , it is sufficient to check all cases with $\delta=0, a>0, t>0$, that is, all pairs $(t, a)$ with $a=30-2 t$ and $1 \leq t \leq 14$. In all cases we take inside $Q$ a general union of $f$ lines of type $(0,1)$ and $S \cup S^{\prime}$ with $\sharp(S)=u, \sharp\left(S^{\prime}\right)=v$, $u=\lfloor(8(8-f)-2(t-f)) / 3\rfloor$, and $3 u+v+2(t-f)=8(8-f)$. To apply [6, Proposition 1] and get $h^{0}\left(\mathscr{I}_{Y}(3)\right) \cdot h^{1}\left(\mathscr{I}_{Y}(3)\right)=0$, it is sufficient to have $(t-f, a-u-v) \neq(2,3)[6$, Proposition 1]; in the exceptional case we would have $h^{0}\left(\mathscr{I}_{Y}(3)\right) \leq 1$ and even $h^{0}\left(\mathscr{I}_{Y}(3)\right) \leq 3$ would be enough.

(d1) Assume that $1 \leq t \leq 4$. Take $t=f$. We have $u \geq v$, $a \geq u+v$.

(d2) Assume that $t=5$. Take $f=4, u=10$, and $v=0$. Since $v=0$, we do not need to check the value of $h^{0}\left(\mathscr{I}_{Y}(3)\right)$.

(d3) Assume that $6 \leq t \leq 14$. Take $f=5$. Since $t-f>0$, we have $h^{1}\left(Q, \mathscr{I}_{2 S \cap Q}(7,2)\right)=0$. Obviously $a \geq u+v$ and $u \geq v$, but in a few cases one of these inequality is an equality (if $t=12$, then $(a, u, v)=(6,3,1)$; if $t=13$, then $(a, u, v)=$ $(4,2,2)$; if $t=14$, then $(a, u, v)=(2,2,0))$.

(e) Assume that $k=8$. Since $\left(\begin{array}{c}11 \\ 3\end{array}\right)=165$, we need to control the following triples $(t, a, \delta):(1,39,0),(2,36,-3)$, $(2,37,1),(3,34,-2),(3,35,2),(4,32,-1),(5,30,0),(6,27$, $-3),(6,28,1),(7,25,-2),(7,26,2),(8,23,-1),(9,21,0)$, $(10,18,3),(10,19,1),(11,16,-2),(11,17,2),(12,14,-1),(13$, $12,0),(14,9,-3),(14,10,1),(15,7,-2),(15,8,2),(16,5,-1)$, and $(17,3,0)$. For all $t \neq 17$, we use the proof of step (a) with the following quintuples $(t, a, \delta, g, u, v)$ (to show that the proof of step (a) works, we only need to check that in all cases $0 \leq g \leq 7, t>g$ (and hence in all cases we may apply $[17,18]$ or $[19$, Corollary 4.6]), $a \geq u+v$, and $u \geq v+\max \{0, \delta\}):(1,39,0,0,26,1),(2,36,-3,25,2)$, $(2,37,1,25,2),(3,34,-2,25,0),(3,35,2,25,0),(4,32,-1$, 
$24,1),(5,30,0,23,2), \quad(6,27,-3,23,0), \quad(6,28,1,23,0), \quad(7$, $25,-2,1,22,1),(7,26,2,1,22,1),(8,23,-1,3,18,0),(9,21$, $0,3,17,1), \quad(10,18,-3,3,13,1),(10,19,1,3,13,1), \quad(11,16$, $-2,5,8,0), \quad(11,17,25,8,0), \quad(12,14,-1,5,7,1), \quad(13,12,0$, $5,6,2),(14,10,-1,5,6,0),(15,7,-2,5,5,1),(15,8,2,5,5,1)$, and $(16,5,-1,6,2,1)$. If $(t, a, \delta)=(17,3,0)$, then we apply the proof of step (b) with $f=6$ and $f^{\prime}=4$.

\section{Conflict of Interests}

The author declares that there is no conflict of interests regarding the publication of this paper.

\section{Acknowledgment}

The author was partially supported by MIUR and GNSAGA of INdAM (Italy).

\section{References}

[1] J. Alexander, "Singularités imposables en position générale aux hypersurfaces de $\mathbb{P}^{n}$," Compositio Mathematica, vol. 68 , no. 3, pp. 305-354, 1988.

[2] J. Alexander and A. Hirschowitz, "Un lemme d'Horace différentiel: application aux singularités hyperquartiques de $\mathbb{P}^{5}$," Journal of Algebraic Geometry, vol. 1, no. 3, pp. 411-426, 1992.

[3] J. Alexander and A. Hirschowitz, "La méthode d'Horace éclatée: application à l'interpolation en degré quatre," Inventiones Mathematicae, vol. 107, no. 3, pp. 585-602, 1992.

[4] M. C. Brambilla and G. Ottaviani, "On the AlexanderHirschowitz theorem," Journal of Pure and Applied Algebra, vol. 212, no. 5, pp. 1229-1251, 2008.

[5] K. A. Chandler, "A brief proof of a maximal rank theorem for generic double points in projective space," Transactions of the American Mathematical Society, vol. 353, no. 5, pp. 1907-1920, 2000.

[6] E. Ballico, "Postulation of general unions of lines and multiplicity two points in $\mathbb{P}^{r}, r \leq 5$," preprint.

[7] E. Ballico, "Postulation of general unions of lines and double points in a higher dimensional projective space," preprint.

[8] R. Hartshorne and A. Hirschowitz, "Droites en position générale dans $\mathbb{P}^{n}$," in Algebraic Geometry, vol. 961 of Lecture Notes in Mathematics, pp. 169-188, Springer, Berlin, Germany, 1982.

[9] E. Carlini, M. V. Catalisano, and A. V. Geramita, "Bipolynomial Hilbert functions," Journal of Algebra, vol. 324, no. 4, pp. 758781, 2010.

[10] E. Ballico, "Postulation of general unions of lines and decorated lines," preprint.

[11] J. Alexander and A. Hirschowitz, "Polynomial interpolation in several variables," Journal of Algebraic Geometry, vol. 4, no. 2, pp. 201-222, 1995.

[12] E. Carlini, M. V. Catalisano, and A. V. Geramita, "3-dimensional sundials," Central European Journal of Mathematics, vol. 9, no. 5, pp. 949-971, 2011.

[13] E. Carlini, M. V. Catalisano, and A. V. Geramita, "Reduced and non-reduced linear spaces: lines and points," http://xxx.tau. ac.il/abs/1308.6796 .

[14] R. Hartshorne, Algebraic Geometry, Springer, New York, NY, USA, 1977.
[15] C. Ciliberto and R. Miranda, "Interpolation on curvilinear schemes," Journal of Algebra, vol. 203, no. 2, pp. 677-678, 1998.

[16] A. Bernardi, M. V. Catalisano, A. Gimigliano, and M. Idà, "Secant varieties to osculating varieties of Veronese embeddings of $\mathbb{P}^{n}$, , Journal of Algebra, vol. 321, no. 3, pp. 982-1004, 2009.

[17] A. Laface, "On linear systems of curves on rational scrolls," Geometriae Dedicata, vol. 90, pp. 127-144, 2002.

[18] A. Laface, "On linear systems of curves on rational scrolls," http://arxiv.org/abs/math/0205271.

[19] E. Arbarello and M. Cornalba, "Footnotes to a paper of Beniamino Segre," Mathematische Annalen, vol. 256, no. 3, pp. 341-362, 1981. 


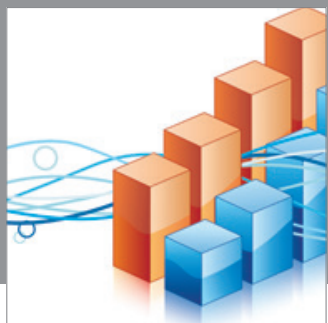

Advances in

Operations Research

mansans

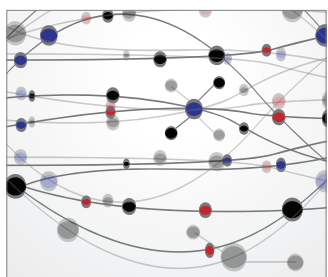

The Scientific World Journal
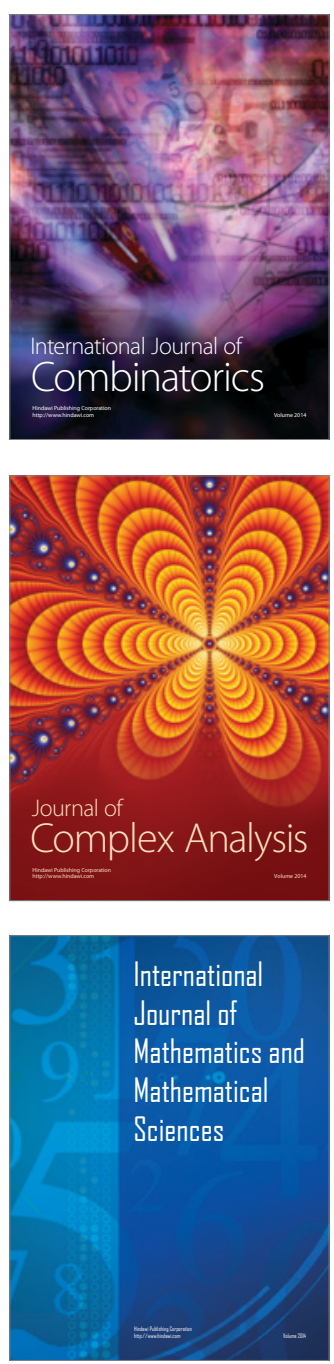
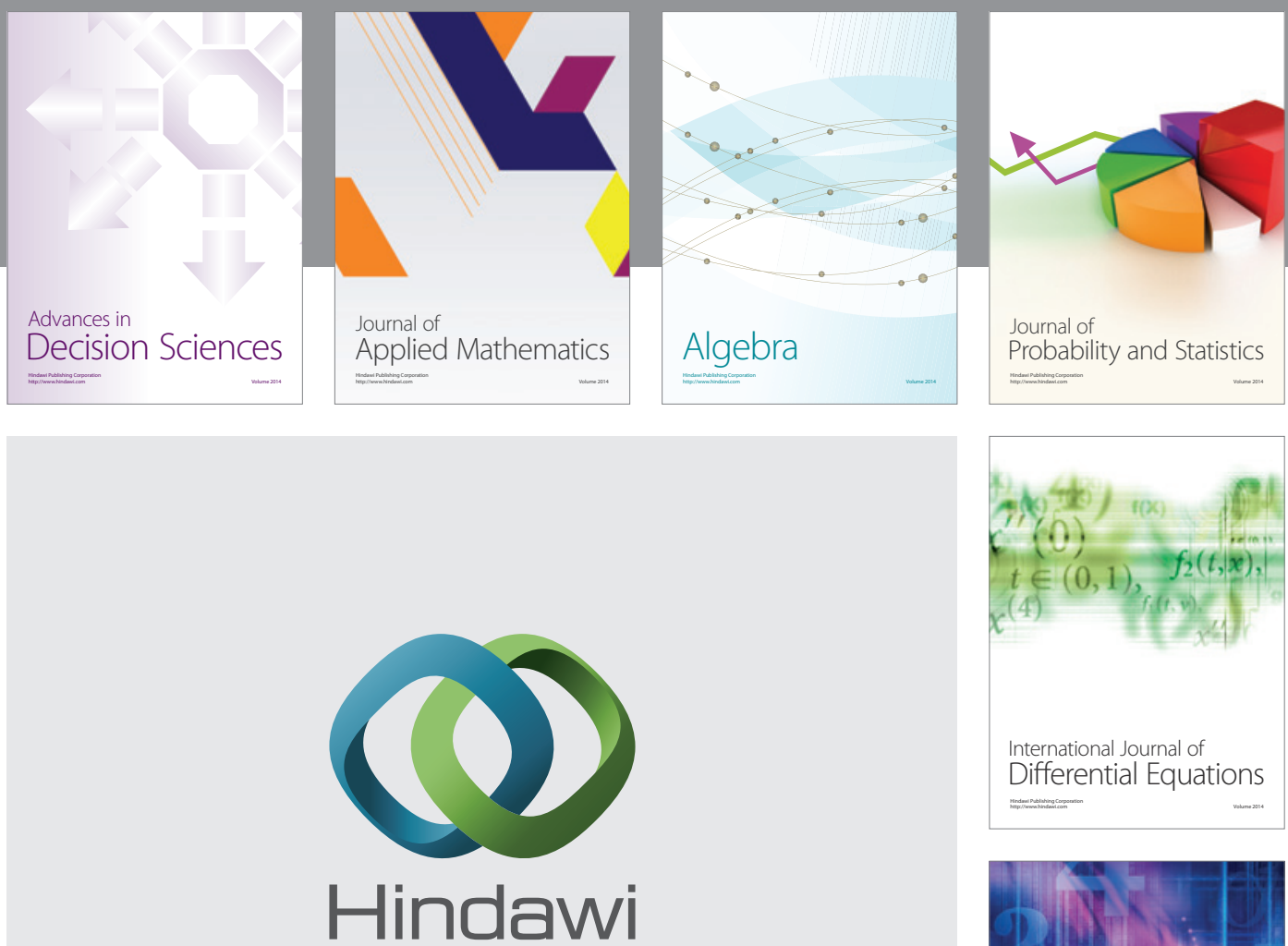

Submit your manuscripts at http://www.hindawi.com
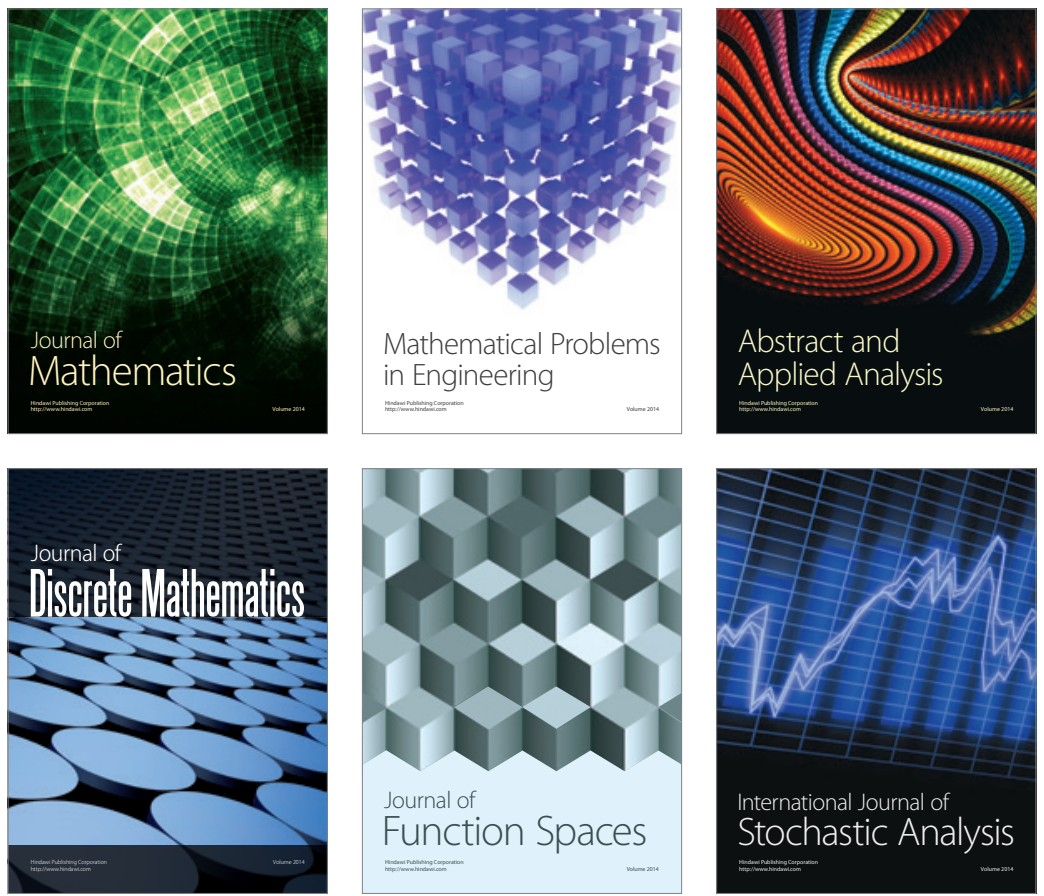

Journal of

Function Spaces

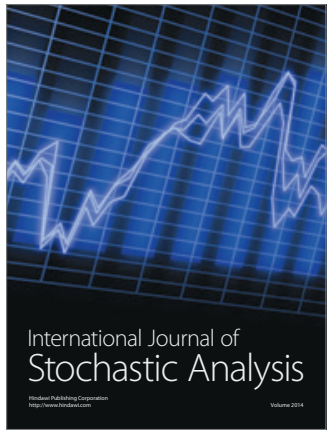

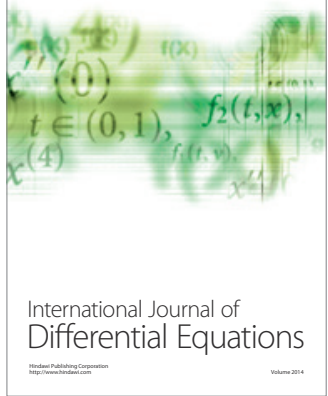
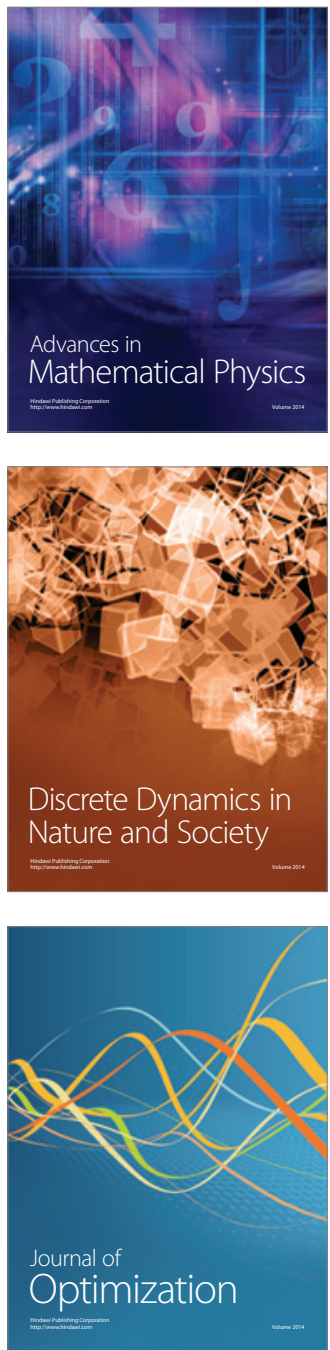